\title{
Status of $R(s)$ measurements by energy scan method
}

\author{
Fedor Ignatov ${ }^{1, a}$ \\ ${ }^{1}$ Budker Institute of Nuclear Physics, SB RAS, Novosibirsk, 630090, Russia
}

\begin{abstract}
The precise low-energy $e^{+} e^{-} \rightarrow$ hadrons cross section are used in SM prediction of various fundamental quantities, like the anomalous magnetic moment of the muon. Direct energy scan experiments were always one of the main tool to provide this information. Status of $R(s)$ measurements using this method is presented, some of the most recent results are discussed in details.
\end{abstract}

\section{Introduction}

The $R(s)$ function is defined as the total hadron production from electron-positrion annihilation normalized to the two muon cross section:

$$
R(s)=\frac{\sigma^{0}\left(e^{+} e^{-} \rightarrow \gamma^{*} \rightarrow \text { hadrons }\right)}{\sigma^{0}\left(e^{+} e^{-} \rightarrow \gamma^{*} \rightarrow \mu^{+} \mu^{-}\right)}
$$

This is one of the fundamental quantities in high energy physics, as it reflects number of quarks and colors $\left(R(s) \sim 3 \sum_{q} e_{q}^{2}\left(1+\delta_{Q C D}(s)\right)\right)$. Measurements of $R(s)$ can be utilized to test perturbative QCD and measure $\alpha_{\mathrm{s}}$ [1]. QCD sum rules provide a method of extracting from the values of $R$ such important parameters as quark masses, quark and gluon condensates, the value of $\Lambda_{\mathrm{QCD}}$ [2]. The total $e^{+} e^{-} \rightarrow$ hadrons cross section gives an input through dispersion integral to the calculation of various fundamental quantities: the running fine structure constant $\alpha_{Q E D}\left(M_{Z}\right)$ [3], hyperfine splitting in muonium [4], the anomalous magnetic moment of the muon $a_{\mu}=\left(g_{\mu}-2\right) / 2[5]$. The $\left(g_{\mu}-2\right) / 2$ value provides a powerful test of the Standard Model with intriguing $3 \div 4$ sigma deviation between the most recent experimental measurement and the theoretical prediction. The main theoretical uncertainty comes from hadronic part of the anomaly, where the value and error are dominated by low energy $R(s)$ below $\sqrt{s}<2 \mathrm{GeV}$ and, particularly, the $e^{+} e^{-} \rightarrow \pi^{+} \pi^{-}$channel gives the biggest contribution (73\% of $a_{\mu}^{\text {had }}$.

The measurements of hadron production at colliders has already celebrated 50 years, starting from first published measurement of $e^{+} e^{-} \rightarrow \rho \rightarrow \pi^{+} \pi^{-}$cross-section (Fig. 1), which was submitted to the journal at 1 September of 1967 [6]. This measurement was performed at the VEPP-2 electron-positron machine in Novosibirsk. In following years the number of different colliders was growing fast by productivity and dimensions, reaching LEP and LHC sizes.

\footnotetext{
a e-mail: ignatov@inp.nsk.su
}

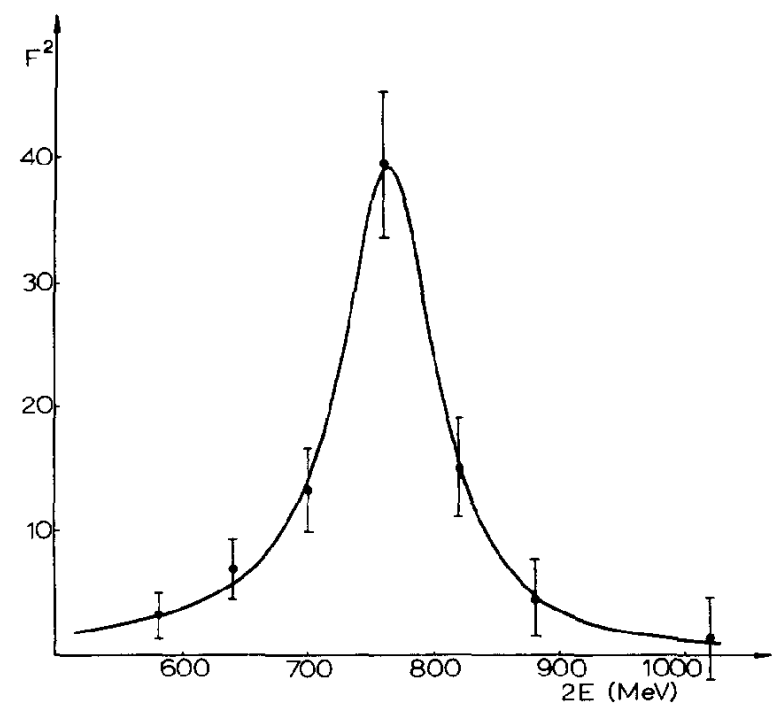

Figure 1. First measurement of hadrons production on colliders. Experimental values of $e^{+} e^{-} \rightarrow \rho \rightarrow \pi^{+} \pi^{-}$cross section by VEPP-2 [6].

In case of the simplest $e^{+} e^{-} \rightarrow \pi^{+} \pi^{-}$channel, many measurements were done over the years coming to current the state of art as can be seen in Fig. 2. The few milestones were along this path: the CMD and OLYA experiments at the VEPP-2M collider performed detail scan of $\rho$ energy region with $2 \%$ systematic precision, to compare to level of $>10 \%$ as was before 1985 . The next breakthrough was improvement of the systematic precision to $0.6 \%$ which was achieved by the CMD-2 experiment [7-10]. The uncertainty in $a_{\mu}^{\text {had }}$ was improved by factor 3 as the result of VEPP-2M measurements, as can be seen in Fig. 3 for $\pi^{+} \pi^{-}$contribution from central $0.6<\sqrt{s}<0.9 \mathrm{GeV}$ energy subrange.

The direct energy scanning approach was somehow limited by statistical precision at that time. After VEPP$2 \mathrm{M}$ results the new developed ISR method [11] at detec- 


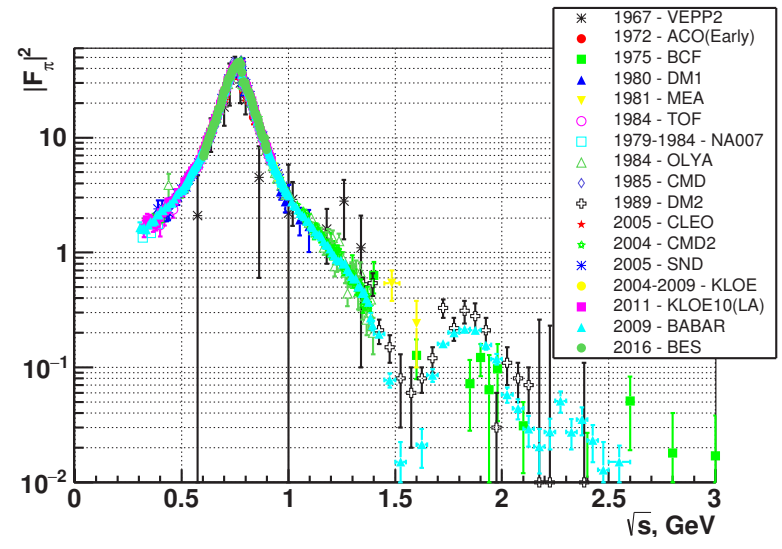

Figure 2. Status of $e^{+} e^{-} \rightarrow \pi^{+} \pi^{-}$cross-section measurement with different experiments contributing over years.

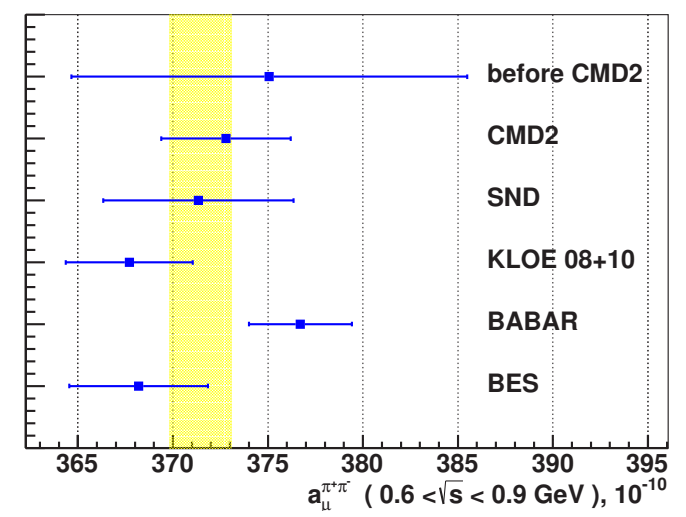

Figure 3. The $\pi^{+} \pi^{-}$contribution to $a_{\mu}^{\text {had }}$ from energy range $0.6<$ $\sqrt{s}<0.9 \mathrm{GeV}$ by most recent experiments.

tors $\mathrm{KLOE}(0.8 \%$ systematic precision) $[12,13]$, BaBar $(0.5 \%)$ [14] and BES (0.9\%) [15] provided successive progress in measurement of this cross-section with less limitations on statistics. Nowadays the $\pi^{+} \pi^{-}$data is statistically dominated by the ISR method with main contribution from KLOE near the $\rho$-meson peak and from BaBar away from $\rho$ (Fig. 4). The most comprehensive analysis with measurements of many various hadronic channels below $2 \mathrm{GeV}$ was performed by the BABAR experiment.

New experiments on the measurement of the muon anomalous magnetic moment in FermiLab [16] and JPARC [17] plan to improve accuracy of the experimental $a_{\mu}$ value by a factor of at least 4 . Precise tests of electroweak theory in experiments at future colliders such as ILC, CLIC, FCC-ee will require knowledge of the hadronic contribution to $\alpha_{Q E D}\left(M_{Z}^{2}\right)$ at the level of $\sim 0.5 \div 0.3 \times 10^{-4}[18,19]$ while the current value is $(276.26 \pm 1.38) \times 10^{-4}$ [5]. To provide comparable accuracies in the theoretical predictions, the accuracy of integral $R$ measurement must be $\sim 0.2 \%$, and particular it is very desirable to improve systematic precision of the $\pi^{+} \pi^{-}$ cross section by at least a factor of two of current level. These days there are three electron-positron colliders do-

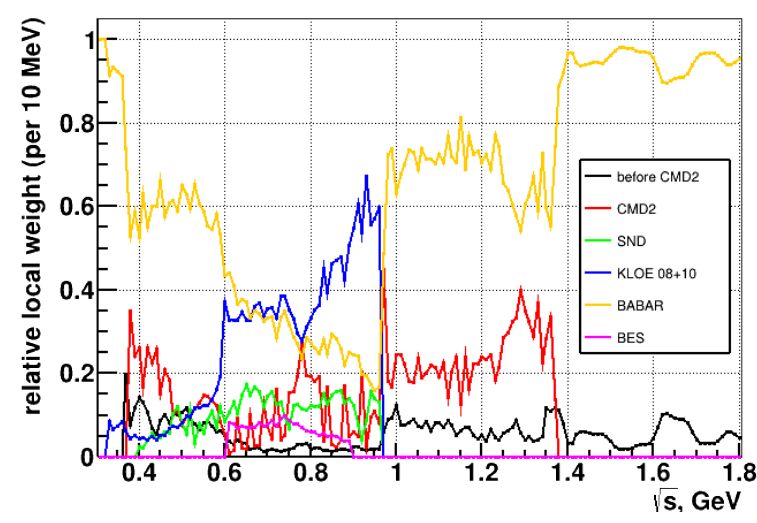

Figure 4. Relative local weight of different experiments to the $\pi^{+} \pi^{-}$cross-section. Nowadays this data is statistically dominated by ISR (KLOE, BaBar).

ing direct exclusive measurement of $\sigma\left(e^{+} e^{-} \rightarrow\right.$ hadrons $)$ : BESIII and KEDR are doing scan of energy from $2 \mathrm{GeV}$ to $5 \mathrm{GeV}$, and the VEPP-2000 is the only one doing scan below $2 \mathrm{GeV}$.

\section{VEPP-2000, CMD-3 and SND}

The electron-positron collider VEPP-2000 [20, 21] has been operating at Budker Institute of Nuclear Physics since 2010. The collider is designed to provide luminosity up to $10^{32} \mathrm{~cm}^{-2} \mathrm{~s}^{-1}$ at the maximum center-of-mass energy $\sqrt{s}=2 \mathrm{GeV}$. The VEPP-2000 is exploiting unique optics with round beams, which allow to reach higher luminosity. During the first 2011-2013 seasons it was collected integrated luminosity comparable to all BaBar effective luminosity from ISR measurements (Fig. 5). The luminosity at energies above $1 \mathrm{GeV}$ was limited by a deficit of positrons and limited energy of the booster. In 2013-2016 the VEPP2000 collider and the detectors have been upgraded. A new injection complex was commissioned at the BINP in 2016. Also the upgrade of the booster ring was done to increase the maximum energy, so it become possible to inject beams to the main ring in the whole available energy range without energy ramping. The VEPP-2000 collider has resumed data taking at the beginning of 2017. Significant improvement in luminosity by a factor of 2 to 8 compared to 2011-2013 was reached as seen in Fig. 6, but there are many things which can gives additional improvements on achievable collider luminosity.

Two detectors CMD-3 [22, 23] and SND [24] are installed at the interaction regions of the collider. In 2010 both experiments started data taking. The physics program [25] includes high precision measurements of the $e^{+} e^{-} \rightarrow$ hadrons cross sections in the wide energy range up to $2 \mathrm{GeV}$, where rich intermediate dynamics involved, studies of known and searches for new vector mesons, studies of $n \bar{n}$ and $p \bar{p}$ production cross sections near threshold and searches for exotic hadrons. It requires a detector with high efficiency for multiparticle events and good en- 
CMD3 collected Luminosity as of 07.07.2017

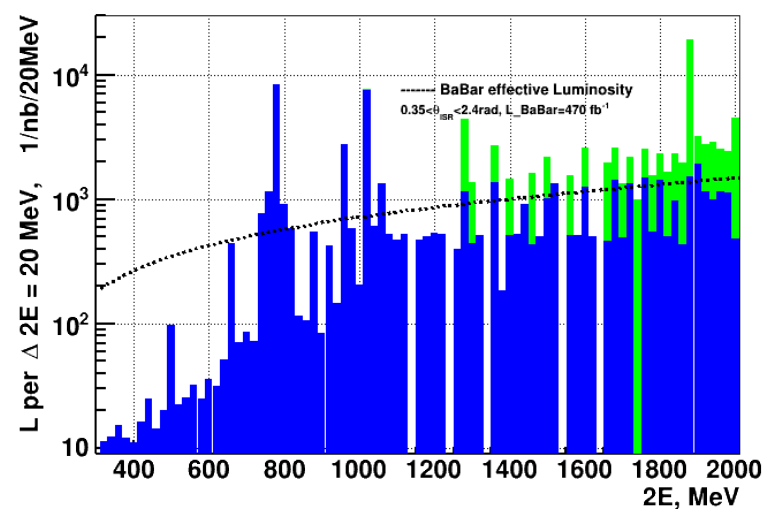

Figure 5. Integrated luminosity collected by the CMD-3 at different energies with $20 \mathrm{MeV}$ energy bins. Blue: 2011-2013 runs, green: 2017 run. The line shows the effective ISR integrated luminosity at BaBar.

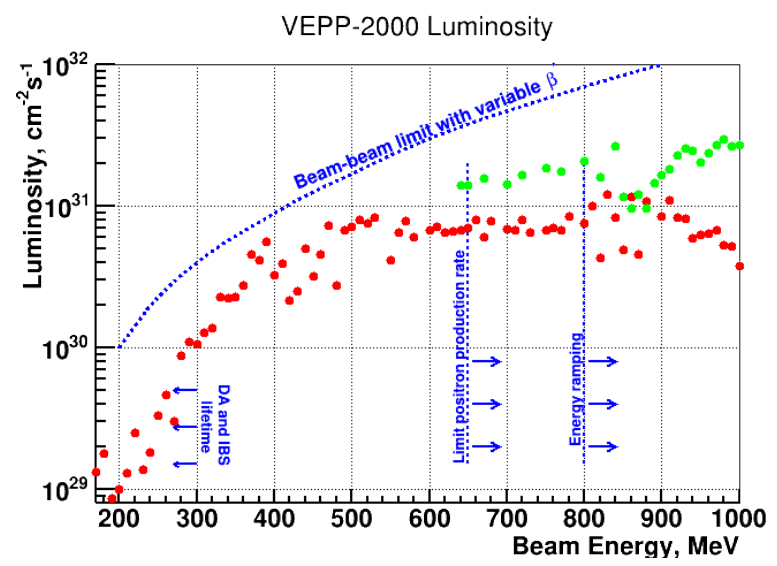

Figure 6. Average luminosity of the VEPP-2000 at different energies. Red points: 2011-2013 runs, green points: 2017 runs.

ergy and angular resolutions for charged particles as well as for photons.

The CMD-3 (Cryogenic Magnetic Detector) is a general-purpose detector, see Fig. 7. Coordinates, angles and momenta of charged particles are measured by the cylindrical drift chamber with a hexagonal cell for uniform reconstruction of tracks. The calorimetry is performed with the endcap BGO calorimeter and the barrel calorimeter. The barrel calorimeter, placed outside of the superconducting solenoid with $1.3 \mathrm{~T}$ magnetic field, consists of two systems: ionization Liquid Xenon calorimeter surrounded by the CsI scintillation calorimeter. The total thickness of the barrel calorimeter is about $13.5 X_{0}$. The LXe calorimeter has seven layers with strip readout which give information about a shower profile and are also able to measure coordinates of photons with about a millimeter precision.

The spherical neutral detector SND is a general purpose nonmagnetic detector, see Fig. 8. The SND consists of the tracking system based on cylindrical drift and proportional chambers placed in a common gas volume,

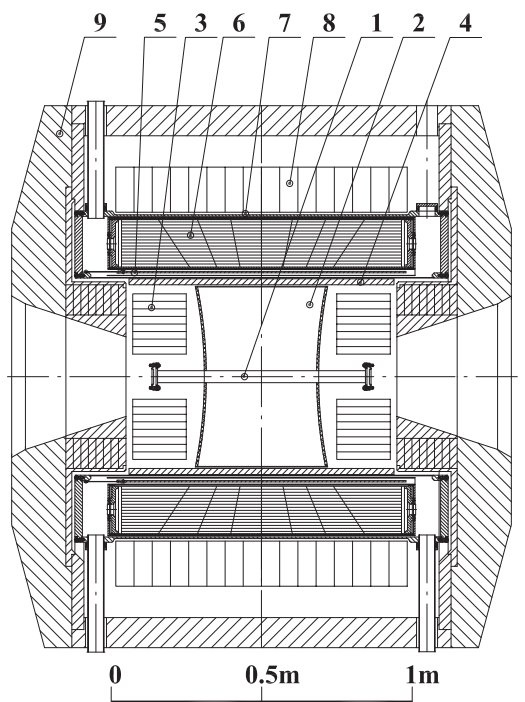

Figure 7. CMD-3 detector: 1 - beam pipe, 2 - drift chamber, 3 - BGO calorimeter, 4 - Z-chamber, 5 - SC solenoid $\left(0.13 X_{0}\right.$, $13 \mathrm{kGs}$ ), 6 - LXe calorimeter, 7 - TOF system, 8 - CsI electromagnetic calorimeter, 9 - yoke, not shown muon range system.

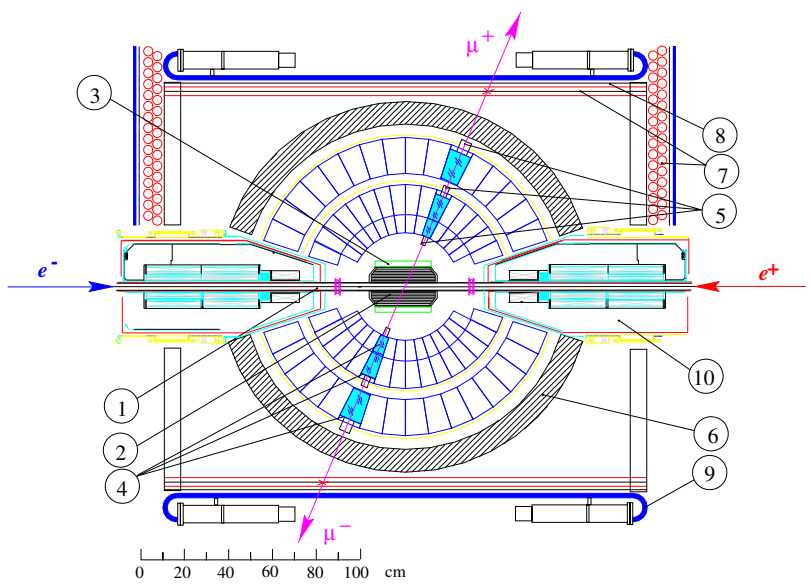

Figure 8. SND detector, section along the beams: 1 - beam pipe, 2 - tracking system, 3 - aerogel Cherenkov counter, 4 - NaI(Tl) crystals, 5 - phototriodes, 6 - iron absorber, 7 - streamer tubes, 8 - iron plates, 9 - scintillation counters, 10 - solenoid of VEPP2000 .

aerogel threshold counters, calorimeter and muon system based on proportional tubes and plastic scintillator. The main part of the SND is the three-layer spherical electromagnetic calorimeter based on $\mathrm{NaI}(\mathrm{Tl})$ crystals. The total calorimeter thickness for particles originating from the interaction region is $13.4 X_{0}$ of $\mathrm{NaI}(\mathrm{Tl})$.

All major channels are under analysis including such as with up to 6 pions or 2 kaons and 2 pions in the final state. The CMD-3 and SND collaborations are already published many results such as: $e^{+} e^{-} \rightarrow \pi^{0} \gamma, n \bar{n}$, $\eta \gamma, \omega \pi^{0}, \eta \pi^{+} \pi^{-}, \pi^{+} \pi^{-} \pi^{0}, \omega \eta, \omega \eta \pi^{0}, K^{+} K^{-}$for SND; $e^{+} e^{-} \rightarrow p \bar{p}, 3\left(\pi^{+} \pi^{-}\right), 2\left(\pi^{+} \pi^{-}\right), \omega \eta, \omega \pi^{+} \pi^{-} \pi^{0}, K^{+} K^{-} \pi^{+} \pi^{-}$, $K^{+} K^{-}, K_{S} K_{L}$ for CMD-3. Many others analyses are in progress, some of them will be discussed below. 


\section{$3 e^{+} e^{-} \rightarrow \pi^{+} \pi^{-}$channel}

The dominant contribution to production of hadrons in the energy range $\sqrt{s}<1 \mathrm{GeV}$ comes from the $e^{+} e^{-} \rightarrow \pi^{+} \pi^{-}$ mode. This channel gives the main contribution to the hadronic term and overall theoretical precision of $a_{\mu}$. It is the most challenging channel due to high precision requirement, which ideally should be known with systematic precision $0.2 \%$ to fulfill requirements of new g-2 experiments and physics on future electron-positron colliders. The CMD-3 has plans to reduce further systematic error which was achieved by the CMD-2. The first energy scan below $1 \mathrm{GeV}$ for the $\pi^{+} \pi^{-}$measurement was performed at VEPP-2000 in 2013. The collected data sample corresponds to about $18 \mathrm{pb}^{-1}$ of integrated luminosity, and it is already higher than in the previous CMD-2 experiment and is similar or better than in the BaBar [14] and the KLOE $[12,13]$ experiments (Fig. 9).

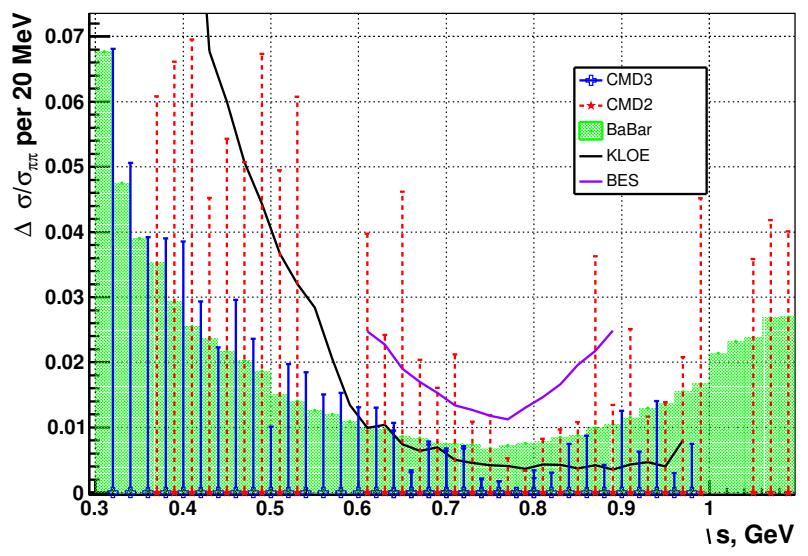

Figure 9. Statistical precision of $\left|F_{\pi}\right|^{2}$ from the CMD-3 data in comparison with CMD-2, BaBar, KLOE and BESIII results.

The crucial pieces of analysis to reach claimed goal includes stable $e / \mu / \pi$ separation, precise fiducial volume determination, theoretical precision of radiative corrections, etc. And important point is that many systematic studies rely on high collected statistics.

The $\pi^{+} \pi^{-}$process has a simple event signature with 2 back-to-back charged particles. They can be selected by using the following criteria: two collinear well reconstructed charged tracks are detected, these tracks are close to the interaction point, both tracks are inside a good region of the drift chamber. The selected data sample includes events with $e^{+} e^{-}, \mu^{+} \mu^{-}, \pi^{+} \pi^{-}$pairs and cosmic muons, and it practically doesn't contain any other physical background at energies $\sqrt{s}<1 \mathrm{GeV}$. These final states can be separated using either the information about energy deposition in the calorimeter or that about particle momenta in the drift chamber. At low energies momentum resolution of the drift chamber is sufficient to separate different types of particles. The pion momentum is well aside from the electron one up to energies $\sqrt{s} \lesssim 0.9 \mathrm{GeV}$, while the $\mu^{+} \mu^{-}$events are separated from others up to $\sqrt{s} \lesssim 0.66 \mathrm{GeV}$. At higher energies the peak of electron

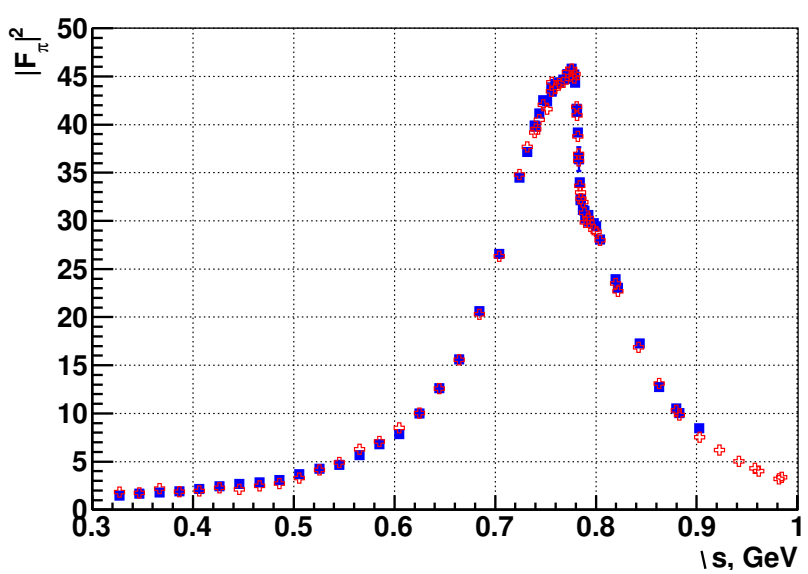

Figure 10. Preliminary results on $F_{\pi}^{2}$ from CMD-3. Open crosses - separation done on the calorimeter information, filled squares - on particle momentum. Some additional corrections, common to two methods (e.g., the trigger efficiency), are not applied.

shower in the calorimeter is far away from the peak of minimal ionization particles. The separation using energy deposition works better at higher energies and becomes less robust at lower energies.

Full energy deposition in combined LXe and CsI calorimeters is used at the moment. A further methods are under development to exploit full power of the layered barrel calorimeter. Additional information from independent measurements of energy deposition in 7 strip layers of the LXe and in the CsI calorimeter gives better discrimination power between different particles due to different interaction process involved (electromagnetic shower, ionization process, nuclear interaction).

The preliminary result on the pion formfactor measured by the CMD-3 is shown in Fig 10 with the comparison of two approaches using either momentum information or energy deposition. The additional corrections, common to two methods (e.g., the trigger efficiency), are not applied. These two methods overlap in the wide energy range and provide a cross-check of each other, allowing to reach in future a systematic error of event separation at the level of $0.2 \%$.

The contribution to systematic precision from $e / \mu / \pi$ separation is estimated now as $0.1-0.5 \%$ when using momentum information and up to $1.5 \%$ in case of by energy deposition, and it should be greatly improved with exploiting of full power of the combined barrel calorimeter.

Determination of the fiducial volume can be made independently with help of the LXe and Z-chamber subsystems. It allows an efficient monitoring of detector operation stability during data taking. The relative difference in scale between this two subsystem was stable at level which corresponds to $0.1 \%$ systematic error of luminosity determination at $\theta_{\text {track }}=1 \mathrm{rad}$.

Measurement of beam energy by Compton backscattering of the laser photons with precision $\sigma_{E}<50$ $\mathrm{keV}$ [26] helps to keep a systematic uncertainty from this source below $0.1 \%$. 
The systematic uncertainty coming from tracks reconstruction inefficiency mostly cancels out in normalization ratio of number of pions to number of electrons. A difference is still present because of the pion specific losses: nuclear interactions and decays in flight. This can be studied in comparisons of data to simulation prediction, and at the moment the agreement is at level $\sim 10 \%$ of the effect, which contributes to total systematic error as $0.6-0.3 \%$. This part will be improved with better drift chamber understanding, which include accounting of detail per drift cell inefficiencies, noise level, etc.

Another important source of systematics is a theoretical precision of radiative corrections [27]. Additional studies like crosschecks of different calculation approaches and further proof from comparison with experimental data are necessary in this field. The high statistics collected with the CMD-3 allowed to observe a discrepancy in momentum distribution of experimental data vs theoretical spectra from the MCGPJ generator[28], which is used in this analysis. The source of the discrepancy was understood: the collinear approximation for photon jets was not good enough to describe differential cross-section in the $P^{+} \times P^{-}$momentum distributions when both final leptons has energy much below initial value (when 2 final photons are involved in kinematics).

Comparison between the MCGPJ[28] and the BabaYaga@NLO[29] generators was performed. The integrated cross-section for the cuts applied is well consistent at the level better than $0.1 \%$ between both tools, and BabaYaga@NLO better describes the data in momentum distribution. While this discrepancy mostly doesn't affect analysis by energy deposition, it becomes crucial if momentum distribution information is used.

Several steps to improve the MCGPJ were done, main one was including of photon jets angular distribution in the generator. The consistency between both generators was improved, but there is still discrepancy at level $\sim 10 \%$ in momentum tails, which gives additional $0.0 \div 0.4 \%$ contribution to systematic uncertainty on number of $\pi^{+} \pi^{-}$ events extracted in analysis based on momentum information. As seen on effects from two photon contributions to momentum spectra, it becomes highly desirable to have exact NNLO $e^{+} e^{-} \rightarrow e^{+} e^{-}(\gamma \gamma)$ generator to reach precision $\lesssim 0.1 \%$.

\section{$4 e^{+} e^{-} \rightarrow K^{+} K^{-}, K_{S} K_{L}$ channels}

The largest contribution to $R(s)$ at $\phi$-resonance comes from 2 kaon production channels. The recent CMD-3 result for $e^{+} e^{-} \rightarrow K_{S} K_{L}$ cross section [30] is shown in Fig. 11. This is the most precise measurement of this cross section with reached $1.8 \%$ systematic uncertainty. The data is very well consistent with previous experiments, and the obtained parameters of $\phi$ resonance are in good agreement with PDG data.

The $e^{+} e^{-} \rightarrow K^{+} K^{-}$channel was recently published by the SND experiment [31] for energies above $1.05 \mathrm{GeV}$, and by the CMD-3 [32] experiment below $1.06 \mathrm{GeV}$ as shown in Fig. 12. The SND measurement agrees with the previ-

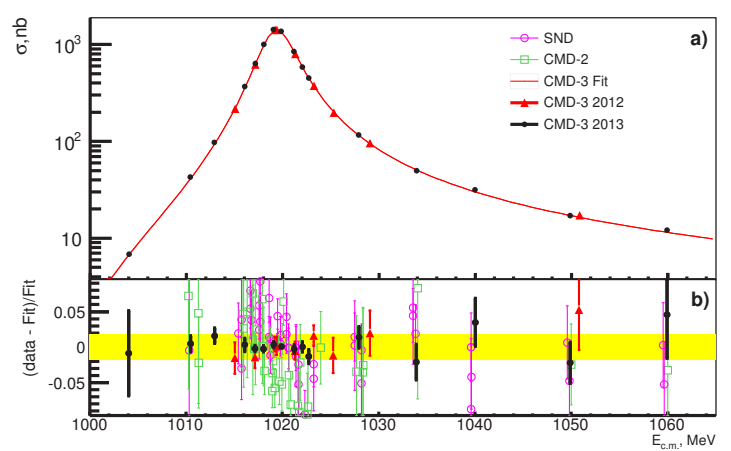

Figure 11. (a) Measured $e^{+} e^{-} \rightarrow K_{S}^{0} K_{L}^{0}$ cross section by CMD-3 experiment, the curve is the fit of data. (b) Relative difference between the data and fit. Comparison between different experimental data is shown. The width of the band shows the systematic uncertainties in CMD-3 experiment.

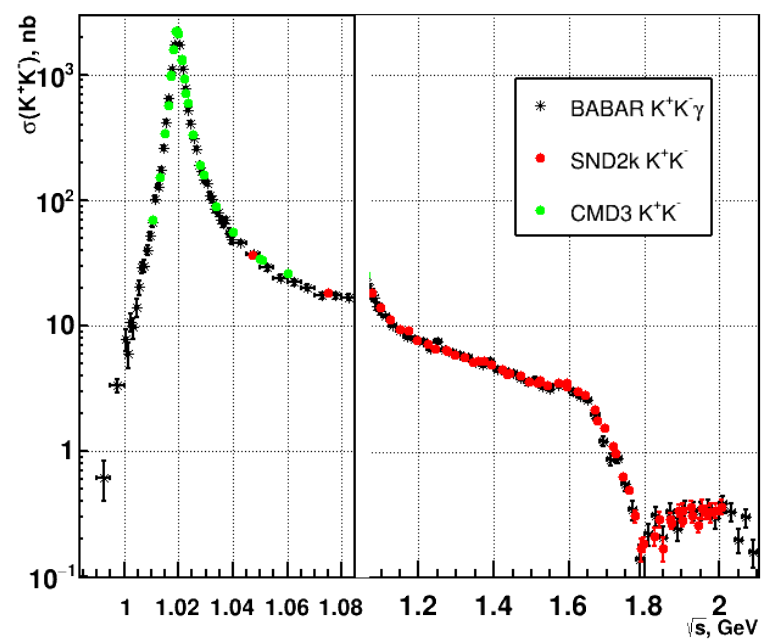

Figure 12. The $e^{+} e^{-} \rightarrow K^{+} K^{-}$cross section measured by CMD3 [32], SND [31] and BABAR [34].

ous most accurate data obtained by the $\mathrm{BaBaR}$ experiment and has comparable or better systematic precision.

The $K^{+} K^{-}$cross section was measured by CMD-3 at the phi-meson energy range with $2 \%$ systematic accuracy. As shown in Figs. 13 and 14, the obtained results have comparable accuracy, but they are not consistent, in general, with the previous data. In particular, an inconsistency between the old CMD-2 [33] result and more recent BaBar data [34] was confirmed. The new CMD-3 measurements of $e^{+} e^{-} \rightarrow K^{+} K^{-}$and $e^{+} e^{-} \rightarrow K_{S} K_{L}$ mention above demonstrates perfect agreement with isospin symmetry: the ratio of coupling constants with the Coulomb factor taken into account is $g_{\phi K^{+} K^{-}} / g_{\phi K_{S} K_{L}} / \sqrt{Z\left(m_{\phi}^{2}\right)}=$ $0.990 \pm 0.017$.

The lower CMD-2 $e^{+} e^{-} \rightarrow K^{+} K^{-}$cross-section is explained by overestimation of the value of the trigger efficiency for slow kaons in the previous experiment. The positive trigger decision of the CMD-2 required the pres- 


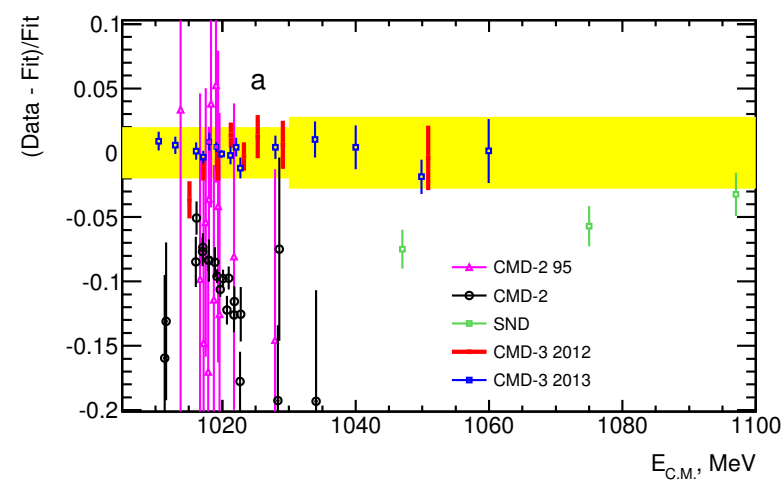

Figure 13. Comparison of the CMD-3 measurement of $e^{+} e^{-} \rightarrow$ $K^{+} K^{-}$to previous CMD-2 [33] and SND [31] measurements. The width of the band shows the systematic uncertainties in CMD-3 study.

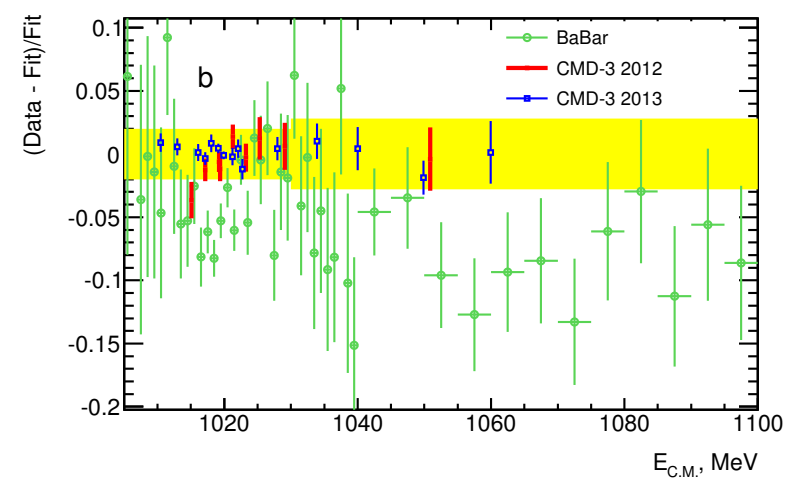

Figure 14. Comparison of the CMD-3 measurement of $e^{+} e^{-} \rightarrow$ $K^{+} K^{-}$to the BaBar measurement [34] using ISR approach. The width of the band shows the systematic uncertainties in the CMD-3 study.

ence of one charged track in the DCh in coincidence with the corresponding hits in the Z-chamber, where the last are not always reached by slow kaons. In contrast to the CMD-2 experiment, the new CMD-3 detector has two independent trigger systems, the Z-chamber is excluded from the decision, and a charged (total) trigger efficiency is close to $100 \%$. The more accurate look on this type of events based on an CMD-3 experience gives a significantly larger trigger efficiency correction than the value obtained in the original CMD-2 analysis [33]. A reanalysis of CMD-2 data is expected.

\section{$5 e^{+} e^{-} \rightarrow \pi^{0} \gamma$ channel}

The cross section of $e^{+} e^{-} \rightarrow \pi^{0} \gamma$ process is the third largest cross section after $e^{+} e^{-} \rightarrow 2 \pi$ and $e^{+} e^{-} \rightarrow 3 \pi$ below $1 \mathrm{GeV}$. The SND group recently published analysis of this channel based on a full data sample collected on the previous VEPP-2M collider [35]. The previous most precise measurements were done by CMD-2 [36] and SND [37] at VEPP-2M, where the SND result was obtained using only $25 \%$ of collected data. The measured

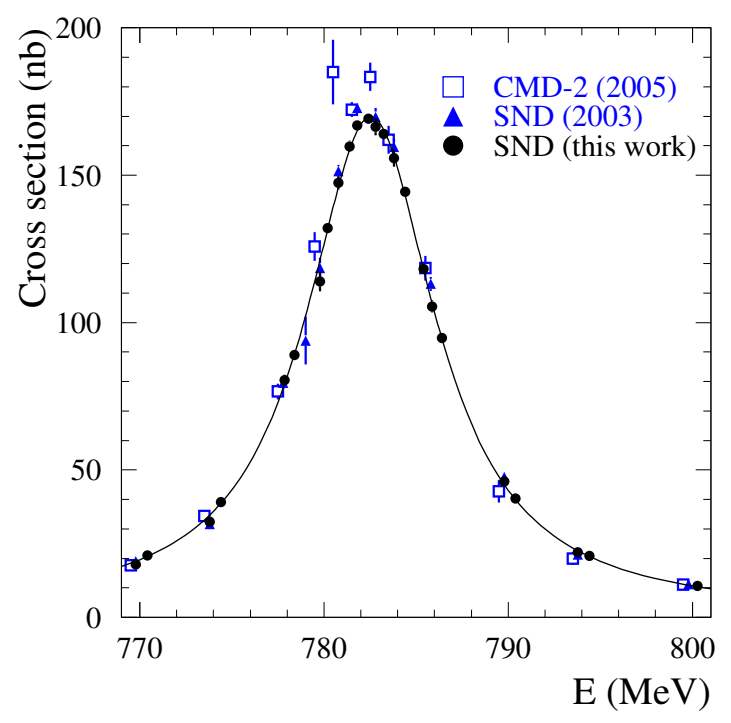

Figure 15. The $e^{+} e^{-} \rightarrow \pi^{0} \gamma$ cross section measured by the SND [35](it is shown only near $\omega$-resonance energy range) together with the previous most accurate measurements by CMD2(2005) [36] and SND(2003) [37]. The curve is VDM fit.

cross section (Fig. 15) is more precise than previous results and agrees with them within the systematic errors. The measurements of the $\rho \rightarrow \pi^{0} \gamma$ and $\omega \rightarrow \pi^{0} \gamma$ branching fractions have better accuracy than PDG values. Using PDG value for $B(\omega \rightarrow 3 \pi) B\left(\omega \rightarrow e^{+} e^{-}\right)$they obtained the ratio $\Gamma\left(\omega \rightarrow \pi^{0} \gamma\right) / \Gamma(\omega \rightarrow 3 \pi)=0.0992 \pm 0.0023$, which is higher than the value reported by KLOE [38] by $3.4 \sigma$.

\section{Unaccounted modes}

The total $e^{+} e^{-} \rightarrow$ hadron cross section below $2 \mathrm{GeV}$ is calculated as a sum of exclusive cross sections for all possible hadronic modes. Some unmeasured channels are calculated using rough isospin relations, which can be very approximative. To fulfill precise $R(s)$ measurement program, it is very important to take into account all possible channels and to do measurements of unaccounted modes or accounted by indirect isospin relations. Some of them were recently studied by the CMD-3 and SND groups.

The process $e^{+} e^{-} \rightarrow \pi^{+} \pi^{-} \pi^{0} \eta$ was studied using $\pi^{+} \pi^{-} 4 \gamma$ final state [39]. This channel can go through the several intermediate states. At least four mechanisms of this reaction: $\omega \eta, \phi \eta, a_{0}(980) \rho$ and structureless $\pi^{+} \pi^{-} \pi^{0} \eta$ were observed. About $50 \%$ of the total cross section at the region below $1.8 \mathrm{GeV}$ is due to the $\omega \eta, \phi \eta$ contributions. Above $1.8 \mathrm{GeV}$ the dominant mechanism of the reaction is $a_{0}(980) \rho$. Only the $\omega \eta$ and $\phi \eta$ contributions were taken into account in total hadron cross-section, while not accounted parts gives about 3-5\% of total $R(s)$ in this energy range. The published measurement by CMD-3 [39] and preliminary SND result on the total $e^{+} e^{-} \rightarrow \pi^{+} \pi^{-} \pi^{0} \eta$ cross section are shown in Fig. 16.

The process $e^{+} e^{-} \rightarrow \omega \pi^{0} \eta$ was studied by the SND in the energy range $\sqrt{s}=1.45 \div 2.00 \mathrm{GeV}$ using fully 


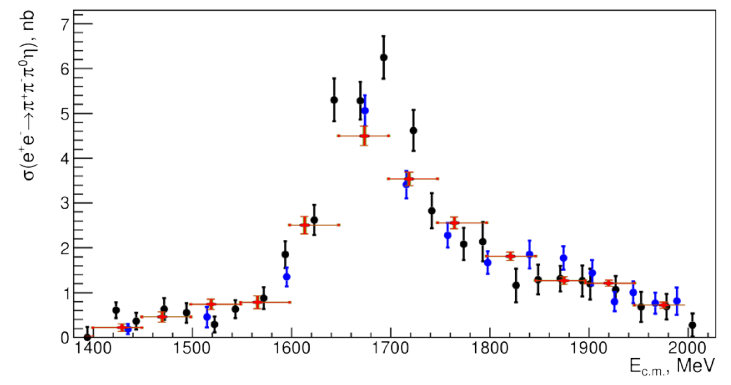

Figure 16. The $e^{+} e^{-} \rightarrow \pi^{+} \pi^{-} \pi^{0} \eta$ cross section measured by CMD3 and SND. Black and blue colors are for the 2011 and 2012 data from CMD-3. Red points - preliminary SND result.

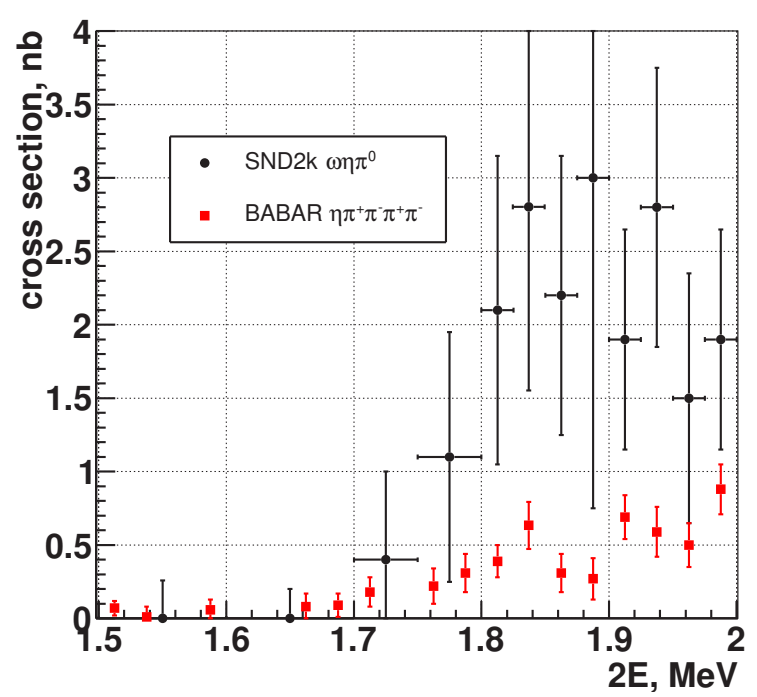

Figure 17. The $e^{+} e^{-} \rightarrow \omega \pi^{0} \eta$ cross section measured by SND [40]. The part of this cross-section was included before in $R(s)$ via "isospin relation" from the $e^{+} e^{-} \rightarrow \eta 2 \pi^{+} 2 \pi^{-}$crosssection measured by BaBar [41], as shown by red points.

neutral $e^{+} e^{-} \rightarrow \pi^{0} \pi^{0} \eta \gamma \rightarrow 7 \gamma$ final state [40]. It is the first measurement of this process. Analysis of the $\pi^{0} \gamma$ invariant mass spectra showed that the $\omega \pi^{0} \eta$ mechanism is dominating in this reaction. From the analysis of the $\eta \pi^{0}$ invariant mass distribution it has been found that the $\omega a_{0}(980)$ intermediate state is dominating in this process. The cross section is about $2.5 \mathrm{nb}$ above $1.8 \mathrm{GeV}$, which takes $5 \%$ of the total hadronic cross section in this energy region. Only part of this cross-section was taken in to account before via "isospin relation" from $e^{+} e^{-} \rightarrow \eta 2 \pi^{+} 2 \pi^{-}$as shown in Fig. 17.

\section{$7 \mathbf{R}(\mathbf{s})$ at $\sqrt{s}>2 \mathrm{GeV}$}

The c.m. energy range from 2 to $5 \mathrm{GeV}$ is almost asymptotic for $u_{-}, d_{-}$, and $s$-quarks. There are no resonances made of light quarks at these energies. The $R$ function has slow dependence with energy from $2 \mathrm{GeV}$ right up to threshold of $D$-mesons $(3.73 \mathrm{GeV})$, except narrow regions around $J / \psi$ and $\psi(2 S)$. The values of $R \approx 2.2$ with current experimental precision are consistent with the pQCD prediction [42]. The 3.73-5.0 GeV energy region are covered by the $c$-quark resonances decayed to $D$-mesons. Numerous $R$ measurements exist in the c.m. energy range between 2 and $5 \mathrm{GeV}$, where many experimental groups studied various states just above the charmonium threshold: Crystal Ball [43], PLUTO [44], DASP [45], MarkI [46], BES [47], KEDR [48, 49]. In general, these measurements are consistent. The most detailed measurement was made by the BESII experiment in total at 165 energy points from 2 to $5 \mathrm{GeV}$ with averaged systematic precision from $7 \%$ to $3.3 \%$. The new BESIII result is expected soon, they did scan of the $2.00-4.59 \mathrm{GeV}$ energy region in 2012-2015 years (125 energy points with the integrated luminosity $1.3 \mathrm{fb}^{-1}$ ). The best systematic precision $2 \%$ was reached by the KEDR experiment, with measurement of $R$ in 20 energy points from 1.84 to $3.72 \mathrm{GeV}$ (Fig. 18). The KEDR experiment has plans to do scan of $2 \mathrm{E}=4.5$ $8(10) \mathrm{GeV}$ energy range, where there is no precise $R(s)$ data consistent with $\mathrm{pQCD}$.

\section{Conclusions}

The precise low-energy $e^{+} e^{-} \rightarrow$ hadrons cross section are used in SM prediction of various fundamental quantities. New precise data are desirable to fulfill requirements of new g-2 experiments and physics on future electronpositron colliders. Direct scan experiments were always providing this $e^{+} e^{-} \rightarrow$ hadrons measurements, while alternative ISR method appeared during last decade. The two methods have owns independent systematic sources, and they are complementary to each other for crosschecking of possible inconsistencies.

The VEPP-2000 is the only collider able to scan energies below $<2 \mathrm{GeV}$ for measurement of exclusive $e^{+} e^{-} \rightarrow$ hadrons channels. A new positron injection complex was commissioned during 2013-2016 years, and the data taking was resumed in 2017 with increased luminosity by few times at $\sqrt{s}>1.2 \mathrm{GeV}$. The first scan below $1 \mathrm{GeV}$ for $\pi^{+} \pi^{-}$measurement was done in 2013. The already collected data sample for the cross section measurement has the same or better statistical precision than was achieved by other experiments. High statistics will allow to study and to control better different systematic contributions, and the improvement here is also expected.

Several previously unmeasured processes like $e^{+} e^{-} \rightarrow$ $\pi^{+} \pi^{-} \pi^{0} \eta$ and $\omega \pi^{0} \eta$ contributed to the total hadronic cross section below $2 \mathrm{GeV}$ has been studied.

New results are expected from CMD-3, SND, KEDR, BESIII with progress forward by luminosity and precision.

This work is supported in part by the Russian Science Foundation (project 14-50-00580) and Russian Foundation of Basic Research (17-02-00847, 17-02-00897).

\section{References}

[1] J. Kühn and M. Steinhauser, Nucl. Phys. B 619 (2001) 588; Erratum-ibid, B640 (2002) 415. 


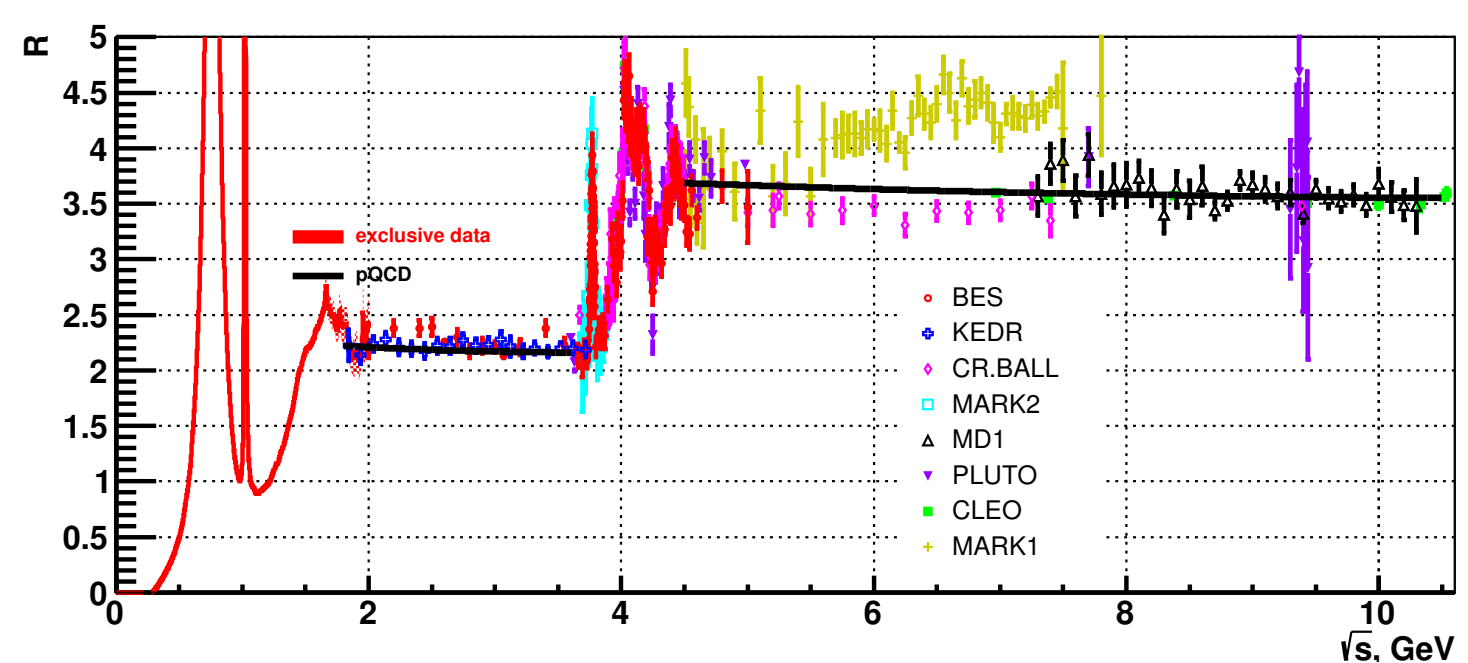

Figure 18. $R=\sigma\left(e^{+} e^{-} \rightarrow\right.$ hadrons $) / \sigma\left(e^{+} e^{-} \rightarrow \mu^{+} \mu^{-}\right)$. Points — the BESII, KEDR and other inclusive data. Shaded area - sum of all exclusive cross-sections for $\sqrt{s}<2 \mathrm{GeV}$. Black line - pCQD prediction.

[2] M.A. Shifman, A.I. Vainshtein and V.I. Zakharov, Nucl. Phys. B 147 (1979) 385.

[3] F. Jegerlehner, Nucl.Phys.Proc.Suppl. 162 (2006): 22

[4] A. Czarnecki, S. I. Eidelman and S. G. Karshenboim, Phys. Rev. D 65 (2002) 053004

[5] K. Hagiwara et al., J. Phys. G 38 (2011): 085003

[6] V. L. Auslander, G. I. Budker, J. N. Pestov, V. A. Sidorov, A. N. Skrinsky and A. G. Khabakhpashev, Phys. Lett. 25B (1967) no.6, 433.

[7] R. R. Akhmetshin et al., Phys. Lett. B 578 (2004):285

[8] V. M. Aulchenko et al.,JETP Lett. 82 (2005) 743

[9] R. R. Akhmetshin et al., JETP Lett. 84 (2006): 413

[10] R. R. Akhmetshin et al., Phys.Lett. B 648 (2007): 28

[11] A. Denig, ISR measurements, this proceedings

[12] F. Ambrosino et al., Phys. Lett. B 670 (2009): 285

[13] F. Ambrosino et al., Phys. Lett. B 700 (2011): 102

[14] J. P. Lees et al., Phys. Rev. D 86 (2012): 032013

[15] M. Ablikim et al., Phys. Lett. B 753 (2016) 629

[16] J. Grange et al., FERMILAB-DESIGN-2014-02, arXiv:1501.06858 [physics.ins-det].

[17] M. Aoki, et al., KEK-J-PARC-PAC2009-12.

[18] M. Baak et al.,Eur. Phys. J. C 74 (2014) 3046

[19] P. Azzi et al., arXiv:1703.01626 [hep-ph].

[20] P. Y. Shatunov et al., Phys. Part. Nucl. Lett. 13 (2016) no.7, 995.

[21] D. Shwartz et al., PoS ICHEP 2016 (2016) 054.

[22] V. M. Aulchenko et al., BUDKER-INP-2001-45

[23] B. Khazin,Nucl. Phys. Proc. Suppl. 181-182 (2008): 376

[24] M. N. Achasov et al., Nucl. Instrum. Meth. A 598 (2009) 31.
[25] S. Eidelman,Nucl.Phys.Proc.Suppl. 162 (2006): 323

[26] E. V. Abakumova et al.,Phys. Rev. Lett. 110 (2013): 140402

[27] S. Actis et al., Eur. Phys. J. C 66 (2010): 585

[28] A. B. Arbuzov et al., Eur. Phys. J. C 46 (2006): 689

[29] G. Balossini et al., Nucl. Phys. B 758 (2006): 227

[30] E. A. Kozyrev et al.,Phys. Lett. B 760 (2016) 314

[31] M. N. Achasov et al., Phys. Rev. D 94 (2016) no.11, 112006

[32] E. A. Kozyrev et al., arXiv:1710.02989 [hep-ex].

[33] R. R. Akhmetshin et al.,Phys. Lett. B 669 (2008) 217

[34] J. P. Lees et al.,Phys. Rev. D 88 (2013) no.3, 032013

[35] M. N. Achasov et al.,Phys. Rev. D 93 (2016) no.9, 092001

[36] R. R. Akhmetshin et al.,Phys. Lett. B 605 (2005) 26

[37] M. N. Achasov et al., Phys. Lett. B 559 (2003) 171

[38] F. Ambrosino et al.,Phys. Lett. B 669 (2008) 223

[39] R. R. Akhmetshin et al.,Phys. Lett. B 773 (2017) 150

[40] M. N. Achasov et al., Phys. Rev. D 94 (2016) no.3, 032010

[41] B. Aubert et al.,Phys. Rev. D 76 (2007) 092005, Erratum: [Phys. Rev. D 77 (2008) 119902]

[42] P. A. Baikov et al., Phys. Lett. B 714, 62 (2012).

[43] A. Osterheld et al., SLAC-PUB-4160, 1986.

[44] J. Burmeister et al., Phys. Lett. 66B (1977) 395.

[45] R. Brandelik et al., Phys. Lett. 76B (1978) 361.

[46] J.L. Siegrist et al., Phys. Rev. D 26 (1982) 969.

[47] M. Ablikim et al.,Phys. Lett. B 677 (2009) 239

[48] V. V. Anashin et al., Phys. Lett. B 753 (2016) 533

[49] V. V. Anashin et al., Phys. Lett. B 770 (2017) 174 